\title{
ÍNDICES DE SITIO PRELIMINARES PARA EUCALIPTO
}

\section{OSCAR GARCIA}

Ingeniero Forestal $\mathrm{Ph}$ D., División Ordenación Forestal e Inventarios. Instituto Forestal Sede Regional X Región, Valdivia.

\section{RESUMEN}

Como parte de un sistema de modelos de crecimiento para eucaliptos en Chile, se desarrolló un modelo de indices de sitio y crecimiento de altura. Los datos provinieron de las parcelas del programa de introducción de especies del Instituto Forestal. Se usó la ecuación de Richards, con estimación simultánea de todos los parámetros por máxima verosimilitud. Debido al pequeño tamaño de las parcelas hubo que idear un nuevo procedimiento para estimar alturas dominantes. Luego de un análisis exhaustivo de la información por especies y regiones, se recomienda provisionalmente un modelo único obtenido con datos de Eucalyptus globulus y $E$. nitens. Aunque estas estimaciones son probablemente lo mejor que se puede esperar con la información existente, ellas presentan limitaciones serias. Para contar en el futura con modelos satisfactorios será necesario establecer y mantener una red adecuada de parcelas permanentes.

Palabras Clave: Indice de Sitio. Eucalyptus.

\section{ABSTRACT}

A site index and height growth model was developed, as a part of a growth modelling system for eucalypts in Chile. Data came from plots of the Instituto Forestal species trials program. Richards' equation was used, with simultaneous maximum likelihood estimation of all the parameters. Because of the small plot size, a new procedure for estimating top heights had to be devised. After an exhaustive analysis by species and geographic regions, for all of them the provisional use of a model obtained with data from Eucalyptus globulus and $\boldsymbol{E}$ nitens is recommended. Although these estimates may approach the best that can be expected with the available data, they have serious limitations. To have satisfactory models in the future it will be necessary to establish and maintain an appropriate network of permanent sample plots.

Keywords: Site Inder, Eucalyptus. 


\section{INTRODUCCIÓN}

Las plantaciones de eucalipto han adquirido una importancia creciente en el sector forestal chileno en los últimos años. La información sobre crecimientos y rendimientos, imprescindible para la evaluación económica, planificación y manejo racional de estas plantaciones, es sin embargo muy limitada.

El proyecto FONDEF 2-33, "Antecedentes Biométricos y Modelos de Apoyo a la Gestión y Manejo Racional del Eucalipto", tiene como objetivos mejorar esta situación. El problema se está abordando en dos frentes. Por un lado, se trabaja en el desarrollo de modelos preliminares utilizando los datos actualmente disponibles. Estos constituirán una primera aproximación, siendo de utilidad inmediata para las empresas forestales y organismos de planificación y guiando la investigación posterior. Al mismo tiempo, se obtienen datos adicionales y se instalan nuevos ensayos y parcelas de muestreo con el fin de mejorar estas herramientas en el futuro.

Los modelos de índice de sitio son el primer componente de un sistema de proyección de crecimiento. Ellos permiten cuantificar la productividad potencial de los terrenos forestales (calidad de sitio) y proyectar el desarrollo en altura. Para eucaliptos en Chile, se han obtenido curvas de indice de sitio para Eucalyptus globulus en Concepción y Arauco (Martinez, 1981; Prado y Barros, 1989). También existen algunos otros estudios para rodales de monte bajo (generados por rebrote) y para localidades restringidas (Prado y Barros, 1989). El objetivo es producir un modelo de alturas actualizado, utilizando al máximo la información existente y usando ecuaciones y técnicas compatibles con los otros componentes del sistema.

Los datos provienen de las parcelas de introducción de especies del Instituto Forestal. Habiendo sido diseñadas con otro propósito, sus características están lejos de ser las ideales para estudios de crecimiento, limitando seriamente la calidad de los resultados. Sin embargo, esta es la única fuente de datos actualmente disponible que cubre rangos de edades y geográficos adecuados. El análisis fustal, a menudo usado en estos casos, aqui no parece factible, al menos en las especies más importantes Eucalyptus globulus y E. nitens, debido a la ausencia de anillos anuales bien definidos.

Otra fuente de incertidumbre es el efecto de las técnicas de establecimiento. En la actualidad se emplean prácticas de preparación de suelos y plantas, fertilización y control de malezas mucho más intensivas que en el pasado. Estas inciden en una mayor sobrevivencia y crecimiento inicial de las plantaciones, aduciéndose en ocasiones que este hecho hace irrelevantes los datos históricos de crecimiento. Hay indicaciones que el efecto podría aproximarse desplazando las curvas horizontalmente en un cierto número de meses dependiendo de los tratamientos, pero el ajuste necesario aún no ha sido cuantificado adecuadamente (García, 1994b). Cabe señalar, sin embargo, que 
presumiblemente en los ensayos aquí utilizados la plantación se efectuó mucho más cuidadosamente que en las plantaciones operacionales de la época y, además, hubo un efecto de selección en los datos al retener solamente las parcelas que tuvieron buena sobrevivencia. En consecuencia, parece plausible que el crecimiento inicial en las parcelas utilizadas no difiera substancialmente del obtenido actualmente con tratamientos intensivos.

En todo caso, es claro que la precisión y exactitud que se pueden esperar del modelo aqui presentado no son altas. Se trata de una primera aproximación, tal vez cercana a lo mejor que se puede conseguir con la información actualmente disponible, pero que deberá revisarse a medida que se obtengan nuevas mediciones.

\section{MATERIAL Y MÉTODO}

\section{Datos}

Los datos utilizados provienen del programa de introducción de especies del Instituto Forestal (INFOR-CORFO. 1986) y fueron extraidos de la base de datos INTROESP (Rojas y Salas. 1984). Además se consideró usar parcelas experimentales del sistema MANPLANT de INFOR. Sin embargo, luego de un proceso de depuración y validación sólo un número muy reducido de éstas resultaron adecuadas, correspondiendo a rodales muy jóvenes. Se prefirió por lo tanto no incluirlas, manteniendo asi un conjunto de datos de características más homogéneas.

Las parcelas del proyecto de introducción de especies son cuadradas, compuestas por 49 plantas espaciadas a $3 \times 3 \mathrm{~m}$, desde la $\mathrm{V}$ Región al norte, y por 100 plantas espaciadas a $2 \times 2 \mathrm{~m}$, desde la VI Región al sur. En ambos casos se midieron los árboles en las 25 posiciones centrales, sirviendo el resto como franjas de aislamiento (INFOR-CORFO, 1986). Es decir, las parcelas medidas tienen superficies de 2250100 $\mathrm{m}^{2}$. En éstas se midió en cada árbol la altura total y el diámetro a la altura del pecho (o el diámetro en la base del árbol en las mediciones iniciales).

Cabe notar que durante el análisis posterior del crecimiento en diámetro y volumen se encontró que, presumiblemente debido a la falta de plantas en el momento de la instalación del ensayo, muchas parcelas tuvieron franjas de aislamiento menores que las especificadas. o inexistentes. Es probable que en el caso de las alturas este hecho no afecte mayormente los resultados, ya que el crecimiento en altura dominante es relativamente poco sensible a las variaciones en la densidad del rodal. Es importante también apreciar que los $100 \mathrm{~m}^{2}$ de la gran mayoría de las parcelas, o aún los $225 \mathrm{~m}^{2}$ de algunas de ellas, es un tamaño muy inferior a lo que seria deseable para el desarrollo de modelos de crecimiento, pudiéndose esperar una alta variabilidad en las observaciones.

Se extrajo de INTROESP la información de Eucalyptus camaldulensis, E. delegatensis. E. globulus, E. nitens, y E. regnans. Esta fue depurada, excluyendo 
mediciones con información incompleta o con errores o anomalías claras. También se eliminaron parcelas con baja sobrevivencia o con muchos árboles dañados y aquellas mediciones iniciales donde se tiene sólo diámetros a la altura del cuello y no a la altura del pecho ${ }^{1}$. De las 752 parcelas y 2.405 mediciones (controles) extraidas inicialmente se retuvieron 239 parcelas con un total de 575 mediciones.

\section{Cuadro $\mathrm{N}^{\circ} 1$}

DATOS

\begin{tabular}{|l|l|c|c|l|}
\hline Especie & Región & Parcelas & Mediciones & UEH \\
\hline E. camaldulensis & Norte & 9 & 21 & $8,9,11$ \\
E. delegatensis & Sur & 88 & 202 & $14,16,19,22,23,25,26,28,29,30$ \\
E. globulus ssp & Norte & 4 & 8 & 11 \\
$\quad$ bicostata & Sur & 5 & 16 & 19,22 \\
E. globulus ssp & Norte & 11 & 23 & $8,9,11$ \\
$\quad$ globulus & Sur & 40 & 92 & $13,14,19,22,28,29$ \\
E. globulus ssp & & & & \\
$\quad$ maidenii & Sur & 3 & 6 & $13,14,19$ \\
E. nitens & Sur & 17 & 44 & $14,16,19,22,23$ \\
E. regnans & Sur & 47 & 119 & $13,14,16,19,22,23,28$ \\
\hline
\end{tabular}

Posteriormente se examinaron gráficamente con mayor detalle las tendencias alturaedad y las relaciones altura-diámetro para cada uno de los controles. Las mediciones que mostraron inconsistencias serias fueron eliminadas.

\section{Método}

Para estimar el modelo se requieren al menos dos mediciones por parcela. En el Cuadro 1 se indica el número de datos finalmente utilizados. La ubicación geográfica de las parcelas se identificó de acuerdo a las Unidades Edafoclimáticas Homogéneas (UEH), obtenidas por intersección de la Clasificación Bioclimática de Di Castri y los Grandes Grupos de Suelos de Roberts y Diaz (INFOR y U. de Chile, 1979; INFORCORFO, 1986; Prado y Barros, 1989). Para este trabajo las UEH se agruparon en una Región Norte, correspondiente a la Región Mediterránea Semiárida de INFOR-CORFO (1986), y una Región Sur, que incluye la Región Mediterránea Central y la Región

${ }^{1}$ Aunque la depuración se hizo con vista al desarrollo de los varios componentes de los modelos de crecimiento, con algunas limitantes las parcelas sin diametros a la altura del pecho podrian también haberse usado para el crecimiento en altura. 
Oceánica de Los Lagos. Esta clasificación se detalla en la Figura $N^{\circ} 1$ y en el Cuadro $\mathrm{N}^{\circ} 2^{2}$.

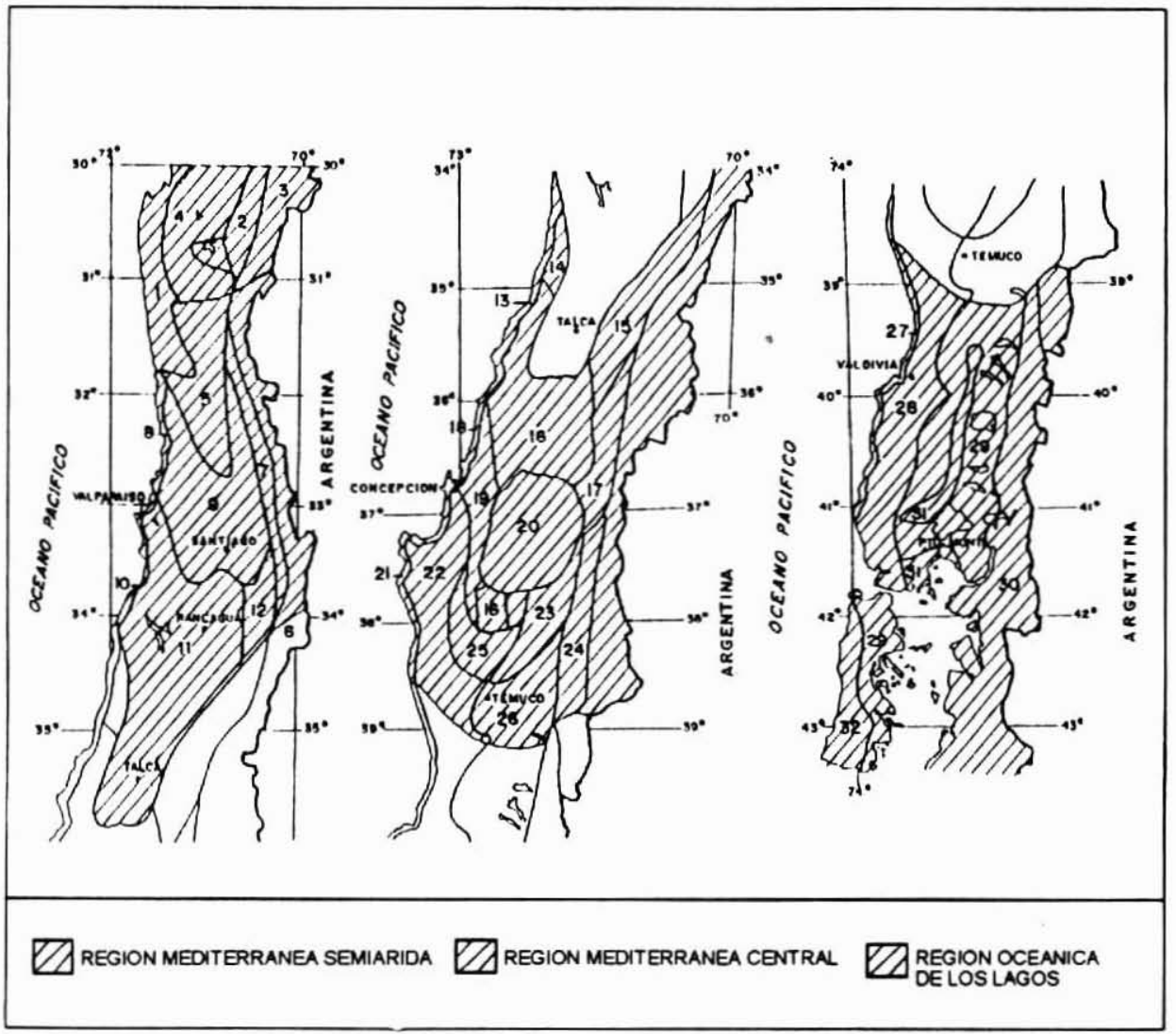

Figura N $N^{0}$ 1. REGIONES EDAFOCLIMÁTICAS

${ }^{2}$ Hay una inconsistencia entre los varios mapas en la asignación de la unidad Paredones. Por simplicidad aqui se la ha incluido en la Región Sur. 
Cuadro $\mathrm{N}^{\circ} 2$

REGIONES EDAFOCLIMÁTICAS HOMOGÉNEAS

\begin{tabular}{|c|c|c|c|}
\hline \multicolumn{4}{|c|}{ Región Mediterránea Semiárida } \\
\hline 1 & Coquimbo & 7 & Procordillera Salamanca - El Yeso \\
\hline 2 & Hurtado & 8 & Zapallar \\
\hline 3 & La L aguna & 9 & Santiago \\
\hline 4 & Ovalle & 10 & Valparaiso \\
\hline 5 & El Choapa & 11 & Rapel-Talca \\
\hline 6 & Cordillera Combartalá-Villarrica & 12 & San José de Maipo \\
\hline \multicolumn{4}{|c|}{ Región Mediterránea Central } \\
\hline 13 & Constitución & 20 & Arenales \\
\hline 14 & Paredones & 21 & Lebu \\
\hline 15 & Molina-Colbuin & 22 & Arauco \\
\hline 16 & Chillán & 23 & Lautaro \\
\hline 17 & Bullileo-Polcura & 24 & Lonquimay \\
\hline 18 & Tomé & 25 & Traiguén \\
\hline 19 & Concepción & 26 & Temuco \\
\hline \multicolumn{4}{|c|}{ Región Oceánica de Los Lagos } \\
\hline 27 & Puerto Saavedra & 30 & Cordillera Sur \\
\hline 28 & Valdivia & 31 & Ñadis \\
\hline 29 & Los Lagos & 32 & Chiloé Poniente \\
\hline
\end{tabular}

\section{Altura Dominante}

Los modelos de crecimiento, y en particular los de indice de sitio, utilizan generalmente alguna medida que representa la altura de los árboles más grandes, llamada altura dominante o altura superior. Esta es preferible a la altura media porque su crecimiento es relativamente insensible. dentro de ciertos rangos, a las diferencias de densidad de rodal. Asi mismo, no es mayormente alterada por los raleos, los que cambian la altura media al remover los árboles de menor tamaño.

Se ha usado una variedad de definiciones de altura dominante. Una de las más comunes y recomendables se basa en un número fijo de los árboles mayores, por ejemplo los 100 mayores por hectárea. En la práctica, si se tiene una parcela de 1/10 o $1 / 20$ de hectárea se eligen los 10 o los 5 árboles mayores en la parcela, respectivamente. La selección puede ser de los árboles más altos o de los de mayor diámetro, y una altura representativa para éstos se puede calcular en una variedad de formas, ya sea con alturas medidas directamente o estimadas con una regresión altura-diámetro.

En realidad, no es dificil darse cuenta que el valor esperado de los 100 árboles mayores en una hectárea es más alto que el del de los 10 mayores en 1/10 o el de los 5 mayores en $1 / 20$. Probablemente las diferencias no son grandes si se usan siempre parcelas de tamaños parecidos. En este caso, sin embargo, la altura del árbol más grande en las parcelas de $100 \mathrm{~m}^{2}(1 / 100$ ha) no sería comparable con alturas dominantes obtenidas de las parcelas de $500 \circ 1000 \mathrm{~m}^{2}$ comúnmente usadas en 
inventarios y recomendadas para parcelas permanentes. Además, una medida basada en un solo árbol tendría una variabilidad bastante alta y no sería muy confiable.

Por estos motivos fue necesario investigar modos de reducir el sesgo debido al tamaño de parcela y la variabilidad de los valores calculados. Brevemente, el procedimiento finalmente adoptado, luego de probar algunas otras alternativas, se puede describir como sigue. Se define la altura dominante como la que correspondería al cuantil $1-45 / N$ de la distribución diamétrica, donde $N$ es el número de árboles por hectárea. Bajo ciertos supuestos, ésta se aproxima a la basada en los 100 mayores por hectárea calculada con parcelas de entre 500 y $1000 \mathrm{~m}^{2}$. Ahora, teniendo esta definición, se necesita un método de estimación. Este consistió en ajustar una distribución Weibull para los diámetros, calcular una regresión altura-diámetro y tomar la altura correspondiente al cuantil apropiado. El método dio buenos resultados considerando el reducido tamaño de las muestras.

\section{Edades}

Los árboles crecen a tasas variables en distintas épocas del año. El crecimiento es mínimo o nulo en invierno, alcanzando un máximo en primavera o verano (en algunos casos la escasez de agua limita el crecimiento en verano, produciéndose dos máximos relativos). Además, el transcurso del crecimiento en altura difiere del crecimiento en diámetro, con este último generalmente prolongándose por más tiempo y alcanzando el máximo más tardíamente.

En consecuencia, para determinar incrementos las parcelas permanentes deberían medirse siempre cerca de una misma fecha, preferiblemente en el periodo de receso o crecimiento minimo para reducir el impacto de las variaciones metereológicas. del desarrollo distinto en altura y diámetro, y de las diferencias en fechas de medición. Esto es particularmente importante con especies de crecimiento rápido, ya que de otro modo habrá una incertidumbre importante en la magnitud efectiva de los intervalos entre mediciones. Es posible introducir ciertas correcciones basadas en información de crecimiento intra-anual (semanal o mensual) (Garcia, 1979), pero, por las razones indicadas, a medida que la fecha de medición se aleja de la de crecimiento mínimo éstas son menos efectivas.

Lamentablemente. las parcelas de introducción de especies han sido remedidas en fechas variadas y. en la mayoría de los casos, en plena temporada de crecimiento. Para paliar en parte este problema se usaron ajustes de edad como en Garcia (1979), basados en el crecimiento intra-anual de pino radiata en Nueva Zelandia (un compromiso entre altura y diámetro), ya que aún no existe información confiable sobre el crecimiento intra-anual de los eucaliptos en Chile. La corrección consistió en sumar los siguientes valores a las edades nominales, según el mes de medición:

$\begin{array}{cccccccccccc}\text { Ene } & \text { Feb } & \text { Mar } & \text { Abr } & \text { May } & \text { Jun } & \text { Jul } & \text { Ago } & \text { Sep } & \text { Oct } & \text { Nov } & \text { Dic } \\ -0.4 & -0.3 & -0,2 & -0.1 & -0,1 & 0.0 & 0.0 & 0,1 & 0,2 & 0.3 & 0,4 & 0,5\end{array}$




\section{Modelos y Estimación de Parámetros}

El crecimiento en altura se representó con la comúnmente usada ecuación de Richards.

$$
H=a\left(1-e^{-b t}\right)^{1 / c}
$$

En esta ecuación. $H$ es la altura dominante y $t$ es la edad. El parámetro $a$ es la asíntota o altura máxima alcanzada por la curva altura-edad, $b$ es un factor que afecta la escala de tiempos (y la tasa de crecimiento para una altura dada) y $c$ determina la altura relativa del punto de inflexión.

En una familia de curvas de índice de sitio uno de estos parámetros (o alguna función de ellos) varia de un rodal a otro de acuerdo a la calidad de sitio, mientras que los otros son constantes comunes a todos los rodales. En lugar de usar directamente el parámetro que varia, se acostumbra a identificar una curva individual por la altura alcanzada a cierta edad clave elegida convencionalmente. La altura a la edad clave es el índice de sitio (García, 1983. 1994a).

Se probaron dos tipos de modelos para los índices de sitio. En el primero, el parámetro que varia con el sitio (parámetro local) fue la asíntota $a$, produciendo curvas "anamórficas", es decir, que difieren entre si por un cambio de escala en el eje $H$. En el segundo tipo de modelo el parámetro local fue $b$, resultando curvas que difieren en la escala de los $t$.

Para estimar los parámetros se utilizó el procedimiento descrito por García (1983). Este se basa en representar la variabilidad de las observaciones por un cierto proceso aleatorio que perturba las tasas de crecimiento, incluyéndose también errores de medición aleatorios. Con este modelo probabilístico se puede calcular la probabilidad que el modelo generara los datos observados para distintos valores de los parámetros, la llamada función de verosimilitud. Las estimaciones de máxima verosimilitud son aquellos valores de los parámetros para los cuáles dicha función toma el valor más alto posible. Este máximo se encuentra con un algoritmo especializado que entrega simultáneamente todos los parámetros, tanto globales como locales.

Este método de estimación es matemática y computacionalmente complejo. Sin embargo, con el software adecuado es relativamente fácil de aplicar y produce estimadores con buenas propiedades, obteniendo el máximo provecho de la información disponible. Esto es especialmente importante en este caso dada la escasez y variabilidad de los datos. De hecho, no está claro como podrían haberse obtenido curvas de indice de sitio usando los métodos más tradicionales. 
El valor de la función de verosimilitud puede usarse en forma aproximada para comparar modelos alternativos y para pruebas de hipótesis. En comparaciones donde el número de parámetros varia, diversas teorías sugieren restar del logaritmo de la verosimilitud (LV) entre 1/2 y 3 unidades por cada parámetro adicional, y considerar como "significativas" diferencias de más de dos unidades luego de este ajuste (García, 1984). Por ejemplo, al combinar dos conjuntos de datos habría 4 parámetros menos que al usar modelos separados (un $a \circ b$ global, $c$ y dos varianzas). Por lo tanto, una diferencia entre la LV agregada y la suma de las LV separadas que excediera algún valor de entre 4 y 14 unidades podría considerarse como evidencia en contra del agrupamiento.

\section{RESULTADOS}

Las posibles diferencias en las relaciones altura-edad entre especies y unidades edafoclimáticas se analizaron exhaustivamente con métodos gráficos y estadísticos. Los datos se graficaron agregándolos de varias maneras y usando colores y transparencias para identificar y contrastar diversos grupos de parcelas. Se ajustaron modelos para distintos conjuntos de datos, comparando los valores obtenidos para los parámetros y la función de verosimilitud. Aquí solamente se pueden`resumir algunos de los aspectos más relevantes.

Botánicamente Eucalyptus globulus y E. nitens están estrechamente relacionados, perteneciendo ambos al grupo denominado "Southern Blue Gums". Del mismo modo, E. delegatensis y E. regnans pertenecen ambos al grupo "Ash".

\section{Eucalyptus globulus y E. nitens}

En la Figura $\mathrm{N}^{\circ} 2$ se muestran los datos para los "Blue Gums", junto con las curvas obtenidas con éstos. El modelo con $b$ como parámetro local produjo un mejor ajuste en este caso, con una asíntota $a=75,3$ metros y $c=0,863$. Los errores estándar estimados para $a$ y $c$ con 8,5 y 0,058 , respectivamente. Con la mayoría de los agrupamientos de datos este tipo de modelo dio la verosimilitud más alta, o en caso contrario un valor cercano al del anamórfico. Como su uso es algo más conveniente para los desarrollos posteriores se ignorarán los modelos ajustados con $a$ local en lo que sigue. 


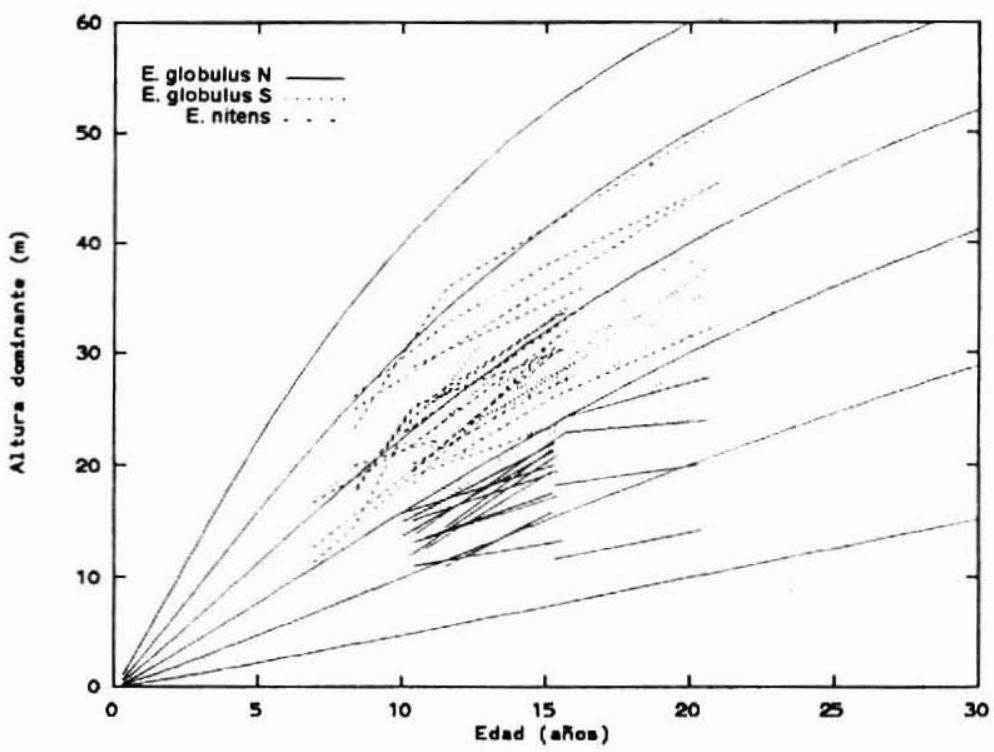

Figura No 2. DATOS Y CURVAS DE fNDICE DE SITIO PARA E. globuluis $y$ E. mitens

Como era de esperar por su origen los datos muestran una muy alta variabilidad. Sin embargo, las curvas parecen representar razonablemente bien la tendencia general y una altura máxima de 75 metros es bastante plausible para estas especies. Las parcelas de la Región Norte presentan generalmente calidades de sitio inferiores, pero no hay desviaciones consistentes de la forma de las curvas del modelo. Usando solamente los datos de la Región Sur se obtiene un modelo muy parecido, con $a=74,4$ y $c=0,849$. Separadamente, la Región Norte da una asíntota de $34,1 \mathrm{~m}$, pero el número de mediciones es bastante exiguo. La diferencia de 6,5 unidades entre las LV (logverosimilitudes) obtenidas combinando o no las regiones no es concluyente.

Al considerar las especies, se nota en la Figura $\mathrm{N}^{\circ} 2$ un cierto número de pares de mediciones en E. globulus. Región Sur, con incrementos altos. Esto hace que al ajustar el modelo usando solamente los datos de esta especie, para la región Sur o para el total, se obtienen asíntotas de 247 y 159 m, respectivamente. La asíntota para E. nitens es de $63 \mathrm{~m}$. El incremento de la LV al separar las dos especies, sin embargo. es de sólo 5,5. La información sobre las sub-especies de E. globulus es demasiado escasa como para indicar diferencias claras.

Se puede concluir que, por el momento, los datos sugieren el uso de un modelo único para ambas especies en el área estudiada. Obviamente, en el futuro una base de datos más extensa y más precisa podrá justificar modelos más especificos. 


\section{Eucalyptus delegatensis}

Se dispone de bastantes datos de esta especie, pero las tendencias altura-edad muestran poca consistencia (Figura $\mathrm{N}^{\circ} 3$ ). Examinando en detalle la información se observó que todos los parámetros muestran gran variabilidad, tanto entre parcelas como dentro de ellas. Generalmente se encuentra un rango muy amplio de diámetros y alturas dentro de cada parcela, con algunos árboles muy pequeños en relación a los árboles mayores. La razón de esto no está clara, tal vez una alta heterogeneidad en el material genético, en la técnica de plantación o una alta sensibilidad de la especie a ésta 0 a las variaciones de micrositio.

El modelo con $b$ local dio $a=83,8$ y $c=0,876$. Como se puede ver en la Figura 3, dentro del rango de los datos las curvas son similares a las obtenidas para los Blue Gums.

\section{Eucalyptus regnans}

Los datos de esta especie se ,muestran en la Figura $\mathrm{N}^{\circ} 4$. Se aprecia bastante variabilidad, con las líneas que representan los incrementos entrecruzándose a menudo. Al graficar las mediciones de altura $v s$. diámetro para cada parcela se observó con frecuencia una forma aplanada en esta relación, sin una tendencia creciente para los árboles mayores. Esto podría deberse a daño apical u otras causas, contribuyendo posiblemente a la variabilidad de las alturas dominantes estimadas.

Con estos datos el procedimiento de estimación no pudo converger a una solución similar a las anteriores. Fijando la asintota $a$ en un valor de 75 metros se obtuvo una solución con $c=0,797$. Las curvas para esta última se muestran en la Figura $\mathrm{N}^{\circ} 4$ junto con las de los Blue Gums. 


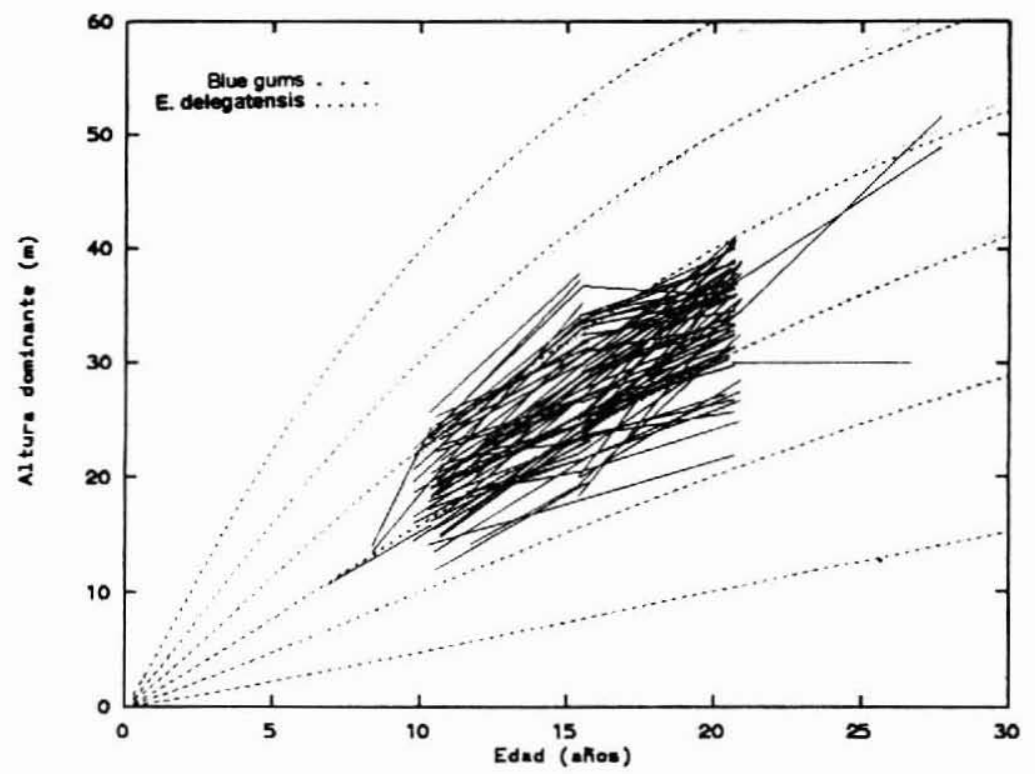

Figura $N^{\circ}$ 3. DATOS DE $E$. delegatensis. (CURVAS OBTENIDAS CON ESTOS DATOS Y CON AQUELLOS DE LOS BLUE GUMS)

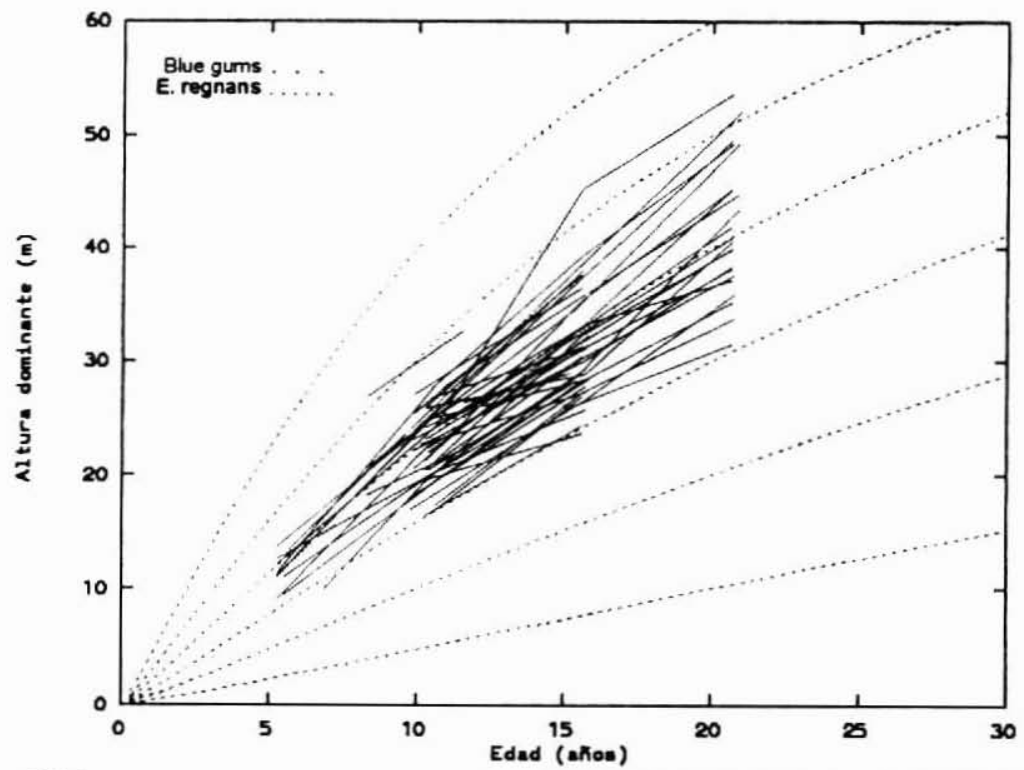

Figura $N^{\circ} 4$. DATOS DE $E$. regnans. (CURVAS OBTENIDAS FI.JANDO $A=75$ COMPARADAS CON LAS DE LOS BLUE GUMS). 


\section{Eucalyptus camaldulensis}

Claramente, la cantidad de datos de esta especie no permite obtener un modelo confiable. Sin embargo, estos no parecen incompatibles con el modelo para los Blur Gums (Figura No 5).

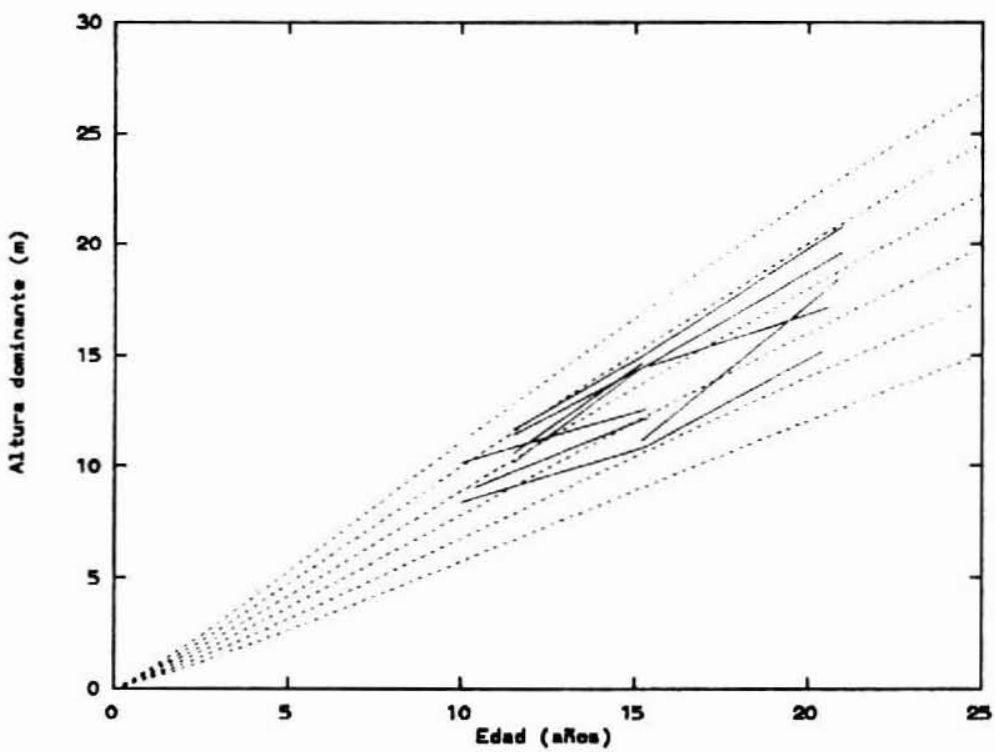

Figura N $N^{\circ}$ 5: DATOS DE E. camaldulensis y CURVAS PARA $E$. globulus $y$ E nitens

\section{Datos Combinados}

Se ajustaron también modelos agrupando la información en varias formas y fijando la asintota a priori a un valor razonable.

Un modelo con todos los datos combinados dio $a=99,1$ y $c=0,930$. Comparado con el uso de dos modelos, uno para los Blue Gums y otro para el resto, la LV disminuye en 6,9 unidades. Las curvas para cada uno de éstos se comparan en la Figura $\mathrm{N}^{\circ} 6$.

La asíntota para el grupo de los " no Blue Gums" resultó muy alta, $174 \mathrm{~m}$, seguramente por la influencia de algunos de los datos de E. regnans. Se repitió entonces el ejercicio del párrafo anterior, pero fijando $a$ en $75 \mathrm{~m}$. Se obtuvieron valores de $c$ de $0,862,0,811$ y 0,828 para "Blue Gums", "no Blue Gums" y el total, respectivamente. La diferencia en $\mathrm{LV}$ fue de 4,5 . Para el total, el fijar la asíntota en 75 $m$ en lugar de dejarla libre redujo la $\mathrm{LV}$ en 3.0 unidades. 


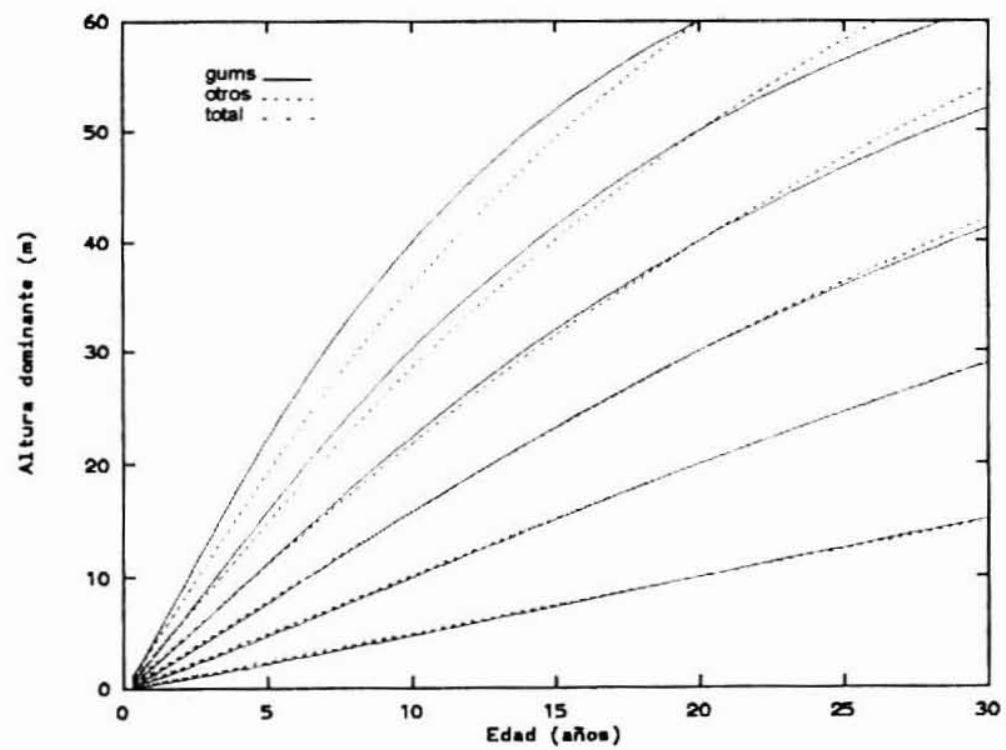

Figura N ${ }^{\circ}$ 6. MODELOS PARA BLUE GUMS, OTRAS ESPECIES Y EL TOTAL DE DATOS

\section{DISCUSIÓN Y CONCLUSIONES}

Aunque la gran variabilidad en los datos disponibles es obvia. es necesario contar con proyecciones de crecimiento por inciertas que éstas sean. Los resultados indican que se puede contar con un modelo aceptable de crecimiento en altura e indices de sitio para ser usado mientras no se disponga de información más confiable.

No se encontró evidencia clara de diferencias importantes en la forma de las tendencias altura-edad entre especies o regiones. Las mayores dudas corresponderian a los sitios de mejor calidad en E. regnans (Figura $\mathrm{N}^{\circ} 4$ ). Sin embargo, la información de E. regnans y la de $\mathbf{E}$. delegatensis es la que presenta mayores inconsistencias e incertidumbre. Considerando además la gran importancia económica que tienen actualmente E. globulus y E. nitens, se recomienda adoptar por el momento para todas las especies de eucalipto el modelo obtenido con los datos de estas últimas.

El modelo de crecimiento en altura es:

$$
H=75,3\left(1-\mathrm{e}^{-\mathrm{bt}}\right)^{1 / 0,863}
$$

Ecuación en la que $H$ es la altura dominante en metros y $t$ es la edad en años. El parámetro $b$ varia con la calidad de sitio. Dada una observación de edad y altura, éste puede ser estimado despejándolo en (2): 


$$
b=-\ln \left[1-(H / 75,3)^{0,863}\right] / t
$$

El indice de sitio $S$ se define como la altura que se alcanzaria a cierta edad indice o clave $t_{s}$. Su relación con $b$ se obtiene por lo tanto substituyendo $H$ y $t$ por $S$ y $t_{\mathrm{s}}$ en (2) o (3). Eliminando $b$, la relación altura-edad en (2) se puede escribir directamente en términos del indice de sitio:

$$
H=75,3\left\{1-\left[1-\left(\begin{array}{ll}
S & 75,3
\end{array}\right)^{0,863}\right]^{t / t_{s}}\right\}^{1 / 0,863}
$$

Igualmente. dada una observación de $H$ y $t$ el índice de sitio estimado es:

$$
S=75,3\left\{1-\left[\begin{array}{ll}
1-\left(\begin{array}{ll}
H & 75,3
\end{array}\right)^{0,863}
\end{array}\right]^{t_{s} t}\right\}^{1 / 0.863}
$$

La edad clave $t_{s}$ es un punto de referencia puramente convencional. Puede elegirse arbitrariamente como cualquier valor conveniente, aunque se acostumbra usar alguna edad algo inferior a las rotaciones usuales para la especie. Para el gráfico de curvas de indice de sitio en la Figura $\mathrm{N}^{\circ} 7$ se selecciona una edad clave de 20 años, superior a las rotaciones preferidas actualmente para eucaliptos pero igual a la edad clave usada para pino radiata en Chile. Conversiones entre indices de sitios para diferentes edades claves pueden hacerse con la ecuación (4).

Es interesante comparar estas curvas con las obtenidas por Martinez (1981) para E. globulus en las zonas de Concepción y Arauco (Figura $\mathrm{N}^{\circ}$ 8). Aunque la forma de las curvas difiere considerablemente, en comparación visual con los datos ambos modelos parecen razonables. con ejemplos de parcelas que se ajustan mejor a las tendencias indicadas por uno u otro de los modelos. Es probable que las nuevas curvas sean algo más confiables. habiendo sido obtenidas con una base de datos más extensa y técnicas más avanzadas. Sin embargo. el hecho que la información disponible no discrimine en forma tajante entre modelos tan diferentes pone de manifiesto la incertidumbre de las proyecciones. Para contar con modelos de crecimiento satisfactorios habrá que aguardar la instalación y remedición de parcelas permanentes en número suficiente y con estándares adecuados para este propósito. 


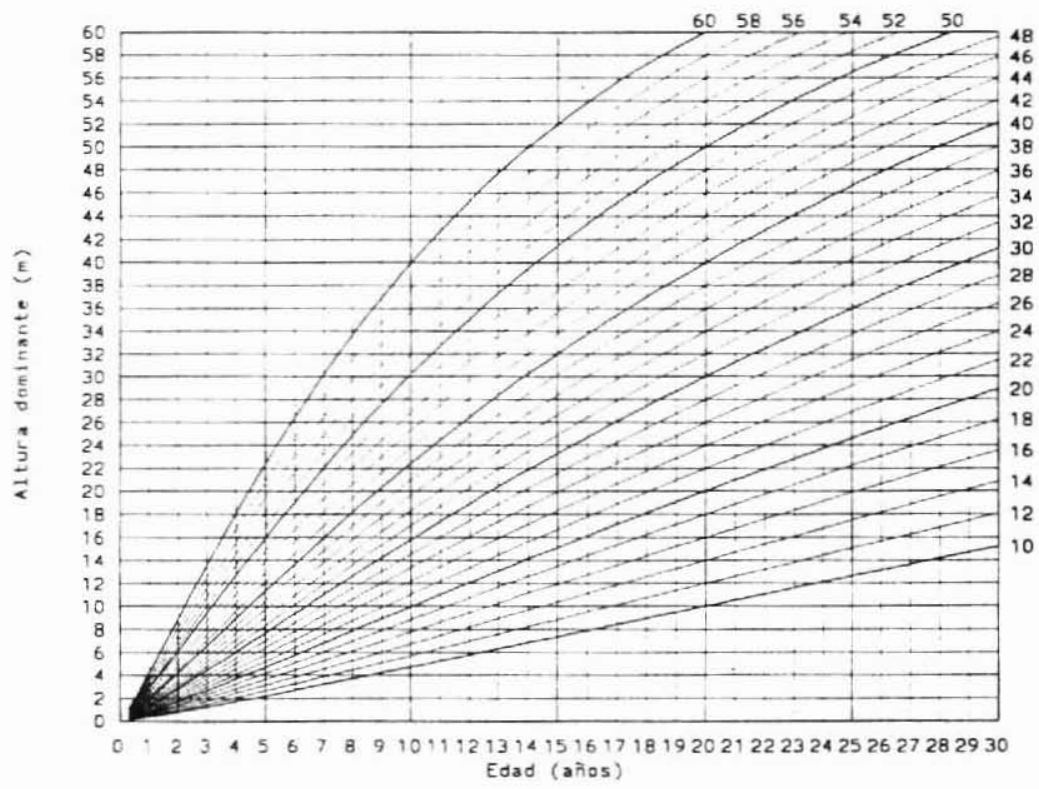

Figura $N^{\circ}$ 7. CURVAS DE INDICE DE SITIO PARA EUCALIPTOS. ECUACIÓN (4), EDAD CLAVE 20 AÑNOS.

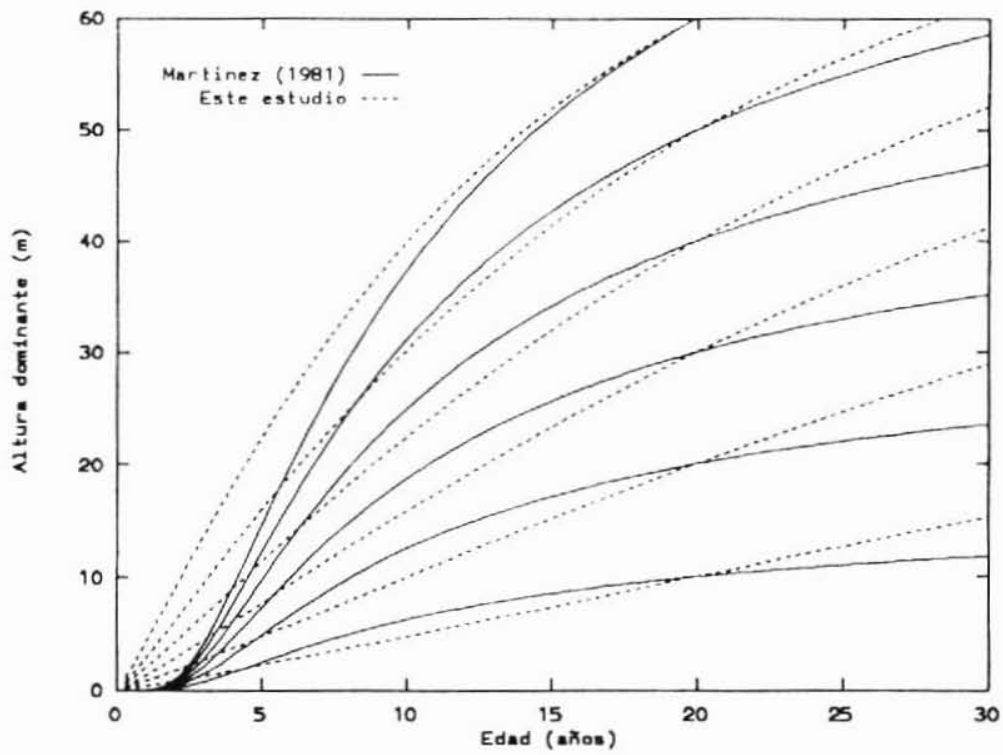

Figura $N^{\circ} 8$. COMPARACIÓN DE CURVAS DE. INDICE DE SITIO 


\section{RECONOCIMIENTOS}

Este trabajo se realizó dentro del proyecto CONICYT-FONDEF 2-33 "Antecedentes Biométricos y Modelos de Apoyo a la Gestión y Manejo Racional del Eucalipto". El Instituto Forestal actúa como unidad ejecutora, con la colaboración de las siguientes empresas: Forestal y Agrícola Monteáguila, Bosques Arauco, Dos Alamos, Forestal A. C. E., Forestal Anchile, Forestal Angol, Forestal Bío Bío, Forestal Cholguán, Forestal Los Lagos, Forestal Millalemu, Forestal Mininco, Forestal Probosque, Forestal Tierra Chilena, Forestal Tornagaleones, Gafonac, Hacienda Chorombo, Inversiones Forestales C. C. A.

\section{REFERENCIAS}

Garcia, O. , (1979). Modelling Stand Development with Stochastic Differential Equations. In Elliott, D.E. (Ed.), Mensuration Systems for Forest Management Planning, pp. 315-334 Forest Research Institute Symposium No. 20. New Zealand Forest Service.

Garcia, O., (1983). A Stochastic Differential Equation Model for the Height Growth of Forest Stands. Biometrics, 39, 1059-1072.

Garcia, O., (1984). New Class of Growth Models for Even-aged Stands: Pinus radiata in Golden Downs Forest. New Zealand Journal of Forestry Science, 14, 65-88.

Garcia, O., (1994a). The State-space Approach in Growth Modelling. Canadian Journal of Forest Research, 24, 1894-1903.

García, O., (1994b). Un Método Simple para Evaluar Técnicas de Establecimiento. En Barros A., S., Prado D., J. A., y Alvear S., C. (Eds.), Actas Simposio Los Eucaliptos en el Desarrollo Forestal de Chile, pp. 295-301. Instituto Forestal, Santiago, Chile.

INFOR, y U. de Chile, (1979). Areas Cubiertas con Ensayos de Introducción de Especies y Ubicación de Nuevas Experiencias. Actividad I.2.2 Introducción de Especies Forestales. Informe II, Provecto CONAF/PNUD/FAO/CHI-76-(003.

INFOR-CORFO, (1986). Especies Forestales Exóticas de Interés Económico para Chile. Gerencia de desarrollo AF 86/32, Instituto Forestal, Corporación de Fomento de la Producción.

Martinez C. A., (1981). Indices de Sitio para Eucalyptus globulus (Lab.) de la Zona de Concepción y Arauco. Tesis para optar al titulo de ingeniero forestal, Universidad de Chile, Escuela de Ciencias Forestales.

Prado D., J. A., y Barros A., S., (1989). Eucalyptus, Principios de Silvicultura y Manejo. Corfo, Gerencia de Desarrollo AF-89/06, Instituto Forestal, Corporación de Fomento de la Producción.

Rojas V., P., y Salas G., G., (1984). Sistema Computacional INTROESP (Introducción de Especies Forestales). Chile Forestal, $N^{\circ} 108,22-23$, Sept. 